\title{
Safety System of W7-X Neutral Beam Injection Heating System
}

\author{
R. Schroeder ${ }^{\mathrm{a}}$, Y. Drider ${ }^{\mathrm{a}}$, D.A. Hartmann ${ }^{\mathrm{a}}$, B. Heinemann ${ }^{\mathrm{b}}$, R. Kairys ${ }^{\mathrm{a}}$, P. McNeely ${ }^{\mathrm{a}}$, R. Riedl ${ }^{\mathrm{b}}$ \\ ${ }^{a}$ Max-Planck-Institut für Plasmaphysik-Teilinstitut Greifswald, Wendelsteinstrasse 1, 17391 Greifswald, Germany \\ ${ }^{b}$ Max-Planck-Institut für Plasmaphysik, Boltzmannstrasse 2, 85748 Garching, Germany
}

In the last experimental campaign (OP1.2b: 7/2018 to 10/2018) of the Wendelstein 7-X stellarator (W7-X), the first of two neutral beam injectors (NBI) was commissioned with 2 plasma sources. The safe operation of the NBI requires the exclusion of hazards to personnel and minimization of system hazards. The NBIcontrol system consists of a standard PLC based on PCS7 from Siemens AG and a separate safety controller based on PNOZmulti from Pilz GmbH. The requirements of the NBI safety control are derived from an analysis of the specific personal and plant hazards in accordance with the international standards IEC 61508 / IEC61511. Particular challenges are the usage of high voltage, open high frequency and high power sub systems, and explosive gases that are distributed over different locations. Firstly, the paper describes the procedure followed by the hazard assessment and for determining solutions for safe operation. Secondly, the paper, describes the hardware structure of the NBI safety system. Lastly details of individual technical solutions, e.g. for access to the high voltage area of the NBI injector, are described.

Keywords: NBI, Plasma Heating, safety control system

\section{Introduction}

The neutral beam injection is one of the plasma heating systems of the Stellerator Wendelstein 7-X. In the last W7-X experimental campaign (OP1.2: 7/2018 to $10 / 2018$ ) one neutral beam injector with two plasma sources was commissioned.

For the next W7-X experimental campaignit is planned to put into operation two injectors each with two plasma sources. In the long term it is planned to equip both injectors with 4 plasma sources each.

The neutral beam injectors are replicas of those installed on the ASDEX Upgrade tokamak, using new technology. All subsystems, power supplies as well as the control and measurement technology, have been newly procured and are state of the art.

The operation of the neutral beam injectors requires the exclusion of hazards to personnel and minimization of system hazards. The basis, for determining the possible hazards, was discussions with the ASDEX Upgrade NBI Group, who have for many years operated a virtually identical neutral particle injection system.

The procedure followed for the hazard assessment and for determining solutions for safe operation of the neutral beam injectors are described in this paper.

The Wendelstein 7-X project has defined regulations and procedures for hazard assessment. The evaluation differentiates between different hazard groups (e.g. mechanical, electrical, thermal hazards, radiation) [1].

The individual hazard groups are classified according to the following criteria [2] (see table 1):

- probability of an accident during the total operating time of the experiment W7-X

- extent of damage to persons, the environment and facilities due to an accident. As an example the classification of damage to persons is given:

$\mathrm{I}=$ death of several persons

$\mathrm{II}=$ irreversible injuries or death of one person

\section{$\mathrm{III}=$ slight injury of one person}

The project has defined three different risk categories so that a clear assignment of a risk class is possible [2]. For each risk class the necessary measures required to minimize the chance and effect of an accident are determined. Typical measures for accident minimization are organizational measures, constructive protective measures and programmable safety controls. Organizational measures are: the definition of personal responsibilities, regular audits, training and instruction. Constructive protective measures are: barriers, enclosures or special mechanical constructions. Safety controllers, actuators and sensors hardly differ from standard hardware in automation technology; however, they are specially designed to ensure the required level of safety and availability for safety-critical systems.

\begin{tabular}{|c|c|c|c|c|}
\hline \multirow[t]{2}{*}{$\begin{array}{l}\text { probability of oc- } \\
\text { currence }\end{array}$} & \multicolumn{4}{|c|}{$\begin{array}{c}\text { extent of damage } \\
\text { (to persons, environment facilities) }\end{array}$} \\
\hline & Level I & Level II & Level III & Level IV \\
\hline $1 / \mathrm{a}<\mathrm{f}$ & 1 & 3 & 6 & 10 \\
\hline $10^{-2} / \mathrm{a}<\mathrm{f}<1 / \mathrm{a}$ & 2 & 5 & 9 & 13 \\
\hline $10^{-4} / \mathrm{a}<\mathrm{f}<10^{-2} / \mathrm{a}$ & 4 & 8 & 12 & 15 \\
\hline $10^{-6} / \mathrm{a}<\mathrm{f}<10^{-4} / \mathrm{a}$ & 7 & 11 & 14 & 16 \\
\hline high risk & \multicolumn{4}{|c|}{$\begin{array}{l}\mathrm{R} \leq 5 \text { : risk cannot be accepted; measures to } \\
\text { significantly reduce risk must be implemented }\end{array}$} \\
\hline medium risk & \multicolumn{4}{|c|}{$6 \leq \mathrm{R} \leq 9$ : risk reduction measures are required } \\
\hline low risk & \multicolumn{4}{|c|}{$10 \leq \mathrm{R} \leq 16$ : can be accepted } \\
\hline \multicolumn{5}{|c|}{$\begin{array}{l}\text { e.g. classification of damage to persons: } \\
\qquad \begin{aligned} \text { I } & =\text { death of several persons } \\
\text { II } & =\text { irreversible injuries or death of one person } \\
\text { III } & =\text { slight injury of one person }\end{aligned}\end{array}$} \\
\hline
\end{tabular}

Table 1: risk matrix of W7X [2]

\section{Safety control system of neutral beam injection}

The NBI safety control system is based on the safety analysis of the NBI system [3]. In this safety analysis, all hazards posed by the NBI system to personnel and components were investigated and, when required, the necessary safety integrity levels (SIL) are defined [4]. 
The safety analysis of the NBI system is based on the standard DIN ISO 12100 [5] valid for machines, which takes into account the European standard 2006/42/EC (Machinery Directive) [6]. The safety controller solutions were designed and implemented on the basis of DIN EN 61511-3 [4].The configurable PNOZmulti 2 control system from the company Pilz GmbH was chosen as the safety control system because of the following features:

- safety-certified hardware and software (up to SIL3)

- a large number of flexible expansion modules

- development software tool with pre-assembled, certified modules for simplified project planning, configuration creation, documentation and troubleshooting

- easy verification \& release of safety program code

- the possibility of a decentralized structure, allowing the distribution among the various NBI locations (torus hall, control room, cooling plant room)

In order to achieve the required safety level that was determined by the hazard assessment, the entire signal chain from the sensor via the controller to the actuator including the architecture of the safety circuit is relevant.
Proof for the technical implementation of the safety level is provided by the probability of failure on demand (PFD) or probability of failure per hour (PFH) from the manufacturer's data of the components used and the hardware fault tolerance (HFT) of the architecture of the safety circuit (from standard DIN EN 61511-3).

The validation of the NBI safety controller requires a series of tests:

1. Check of the program code of the safety control by a second person

2. NBI internal functional test of all safety functions with test protocol

3. Function test by W7-X-Quality Management (QM) for all safety functions with test protocol

Each test protocol must contain the current security check sum of the program so that changes can be detected at any time. Each subsequent program code change requires both detailed documentation of the changes and the re-running all tests.

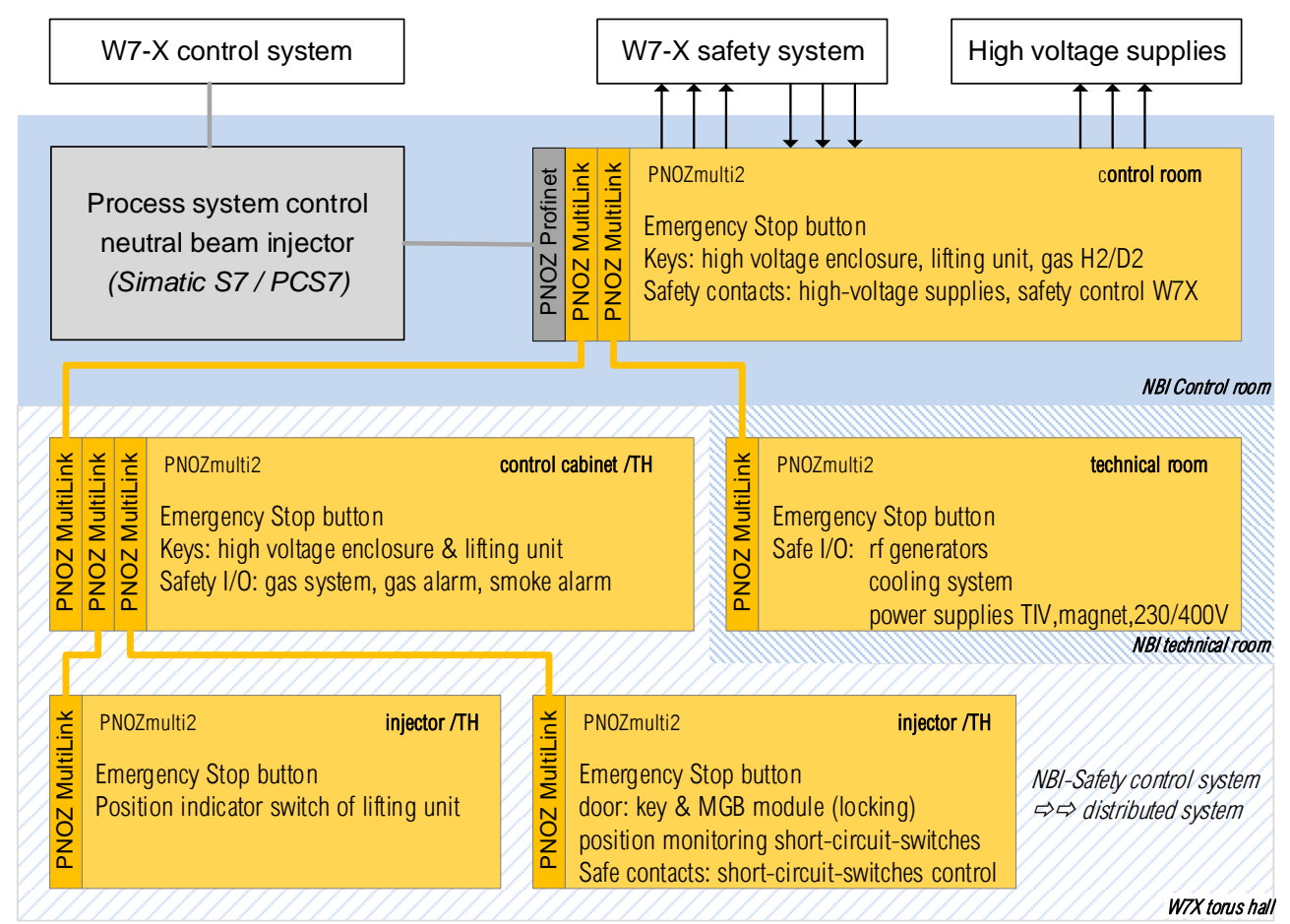

Fig. 1. hardware structure of NBI safety control system

\section{NBI safety control: realization of personnel and system safety}

Both injectors and their controls are identical and are designed for independent operation. However, the cooling system, which was designed for 4 plasma sources of one injectors, is shared by both injectors for cost reasons.

For this reason the paper will only detail the system of a single injector.

The NBI system has a large number of potential hazards (see [3]), in particular with regard to
- personnel hazards:

High voltage (up to $100 \mathrm{KV}$ ), radio frequency generator $(1 \mathrm{MHZ}$ with $150 \mathrm{KW})$ gas $\left(\mathrm{H}_{2} / \mathrm{D}_{2}\right)$

NBI cooling water system (up to 16bar) radiation (X-rays, gamma, neutrons)

- equipment hazard:

$\mathrm{H}_{2} / \mathrm{D}_{2}$ beam (up to $5 \mathrm{MW}$ of beam power) 


\subsection{Fast Interlock System}

Since the NBI safety control system has a reaction time less than 50 of milliseconds, an additional fast safety-relevant NBI interlock system has been implemented to act on the high-voltage system. This interlock system is based on fast hardware logic gates $(<100 \mu \mathrm{s})$. Based on signals from: the W7-X interlock, fast thermal interlock, beam blocking detection, injector interlock it can block the high voltage control signals, thus terminating the NBI beams within a few milliseconds. The NBI interlock system cannot be assigned a safety level due to the hardware used.

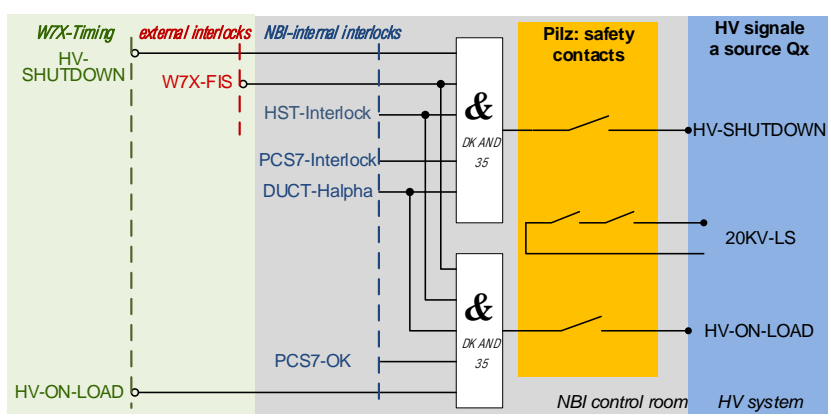

Fig. 2. Fast interlock structure of NBI

\subsection{Safety signals between W7-X and NBI}

The communication between the local NBI safety controller (ISS) and the central experiment controller W7-X (cSS) is done via defined signals. The number of signals was minimized by means of Model-Based Systems Engineering (MBSE, software tools Magic Draw). The signals are implemented as relay contacts, which are designed as either single-channel (SIL1) or dual-channel (SIL2) depending on the safety requirements.

\subsection{NBI-Emergency-Stop (general)}

The NBI system has been designed so that a power failure poses no threat to either personnel or the injector system. In the case of power failure, the components end up in a safe condition automatically, e.g. via spring-closing valves, flaps, slide or gravity falling short-circuit switches. The design of the system in this manner allows a complete shutdown of all power supplies of the NBI system components in the event of a dangerous situation (NBI-EMERGENCY STOP).

The NBI EMERGENCY STOP acts on the main switches of the low voltage power supply, on the output contactors of the NBI control cabinets and on the EMERGENCY STOP inputs of the individual NBI system components. The NBI EMERGENCY OFF is triggered by:

- NBI-EMERGENCY STOP buttons, which are installed system-wide

- W7-X EMERGENCY STOP request from W7-X

- Smoke or a dangerous level of hydrogen gas inside the HV cabin

\subsection{Danger of high voltage}

Generation of the ion beams requires high voltages (HV) of up to $100 \mathrm{kV}$.

The high voltage for each NBI source is supplied from the central high voltage supply of the W7-X project over approximately $100 \mathrm{~m}$ long cables. The cables terminate in the W7-X torus hall in the NBI high-voltage cabin. In this cabin all high-voltage components of the NBI (snubbers, matching resistors, voltage dividers) including the source connections are located. With regard to personnel hazards, the high-voltage cabin was classified as a high risk area. Safety is ensured by a combination of various measures described in the following section.

\subsubsection{Organizational activities}

The high voltage cabin is a restricted area that may only be entered when accompanied by trained NBI staff [7]. Entering and working in the HV cabin required undergoing special training [8]. The entry into the HV cabin is only permitted after approval by a designated person who is qualified by specific training and experience (NBIEFK).

The NBI-EFK is responsible for ensuring that the components in the HV cabin are safely de-energized. This state is guaranteed if:

- the high-voltage system W7-X is not authorized for operation, i.e. the HV outputs in the HGV-W7-X are offline and safety grounded,

- all HV components in the NBI cabin are discharged and grounded.

\subsubsection{Constructive safeguarding}

The walls of the high voltage cabin are made of aluminum sheet $(1.5 \mathrm{~mm})$ and designed to be "light tight", minimizing all gaps and holes. The exterior walls of the HV cabin are extensively grounded so that when the door is closed there is a $100 \%$ personnel safety in relation to the high voltage potentials present within. The door of high voltage cabin can also be locked with a padlock.

\subsubsection{NBI safety control system}

A part of the safety control system is the status-dependent locking of the HV cabin entrance door, based on:

- key transfer system for the HV cabin door

The injector has identical safety locks (one key for both locks) one in the NBI control room and one on the HV cabin. The locks are integrated into the safety control via redundant relay contacts and are part of the logic for the HV cabin door,

- locking of the high-voltage cabin door with a special door module integrated in the safety control (MGB, Euchner company),

- authorization for operation with high voltage,

- position monitoring of the source-specific short-circuitswitches in the HV cabin,

- optical signaling of the hazard potential in the cabin by external LED lights:

green: all short-circuit-switches are safely closed red: NOT all short-circuit-switches are safely closed 


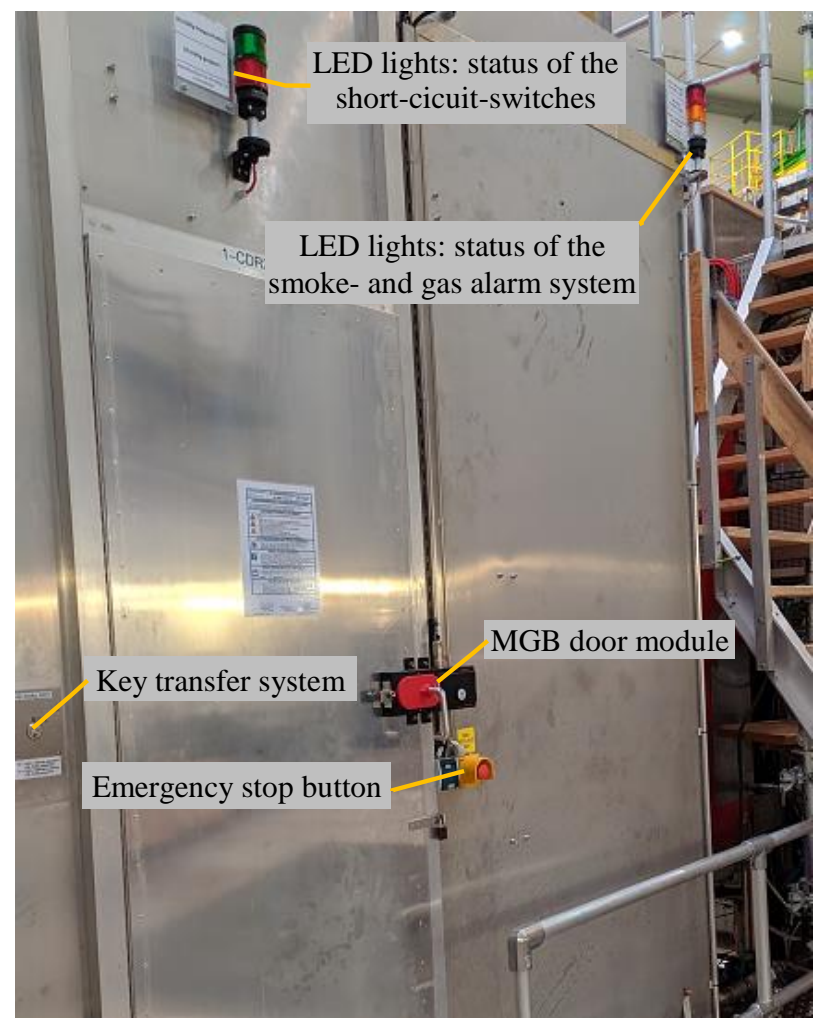

Fig. 2. High voltage cabin incl. MGB door module

Examples of safety logic implemented in the NBI safety controller on the basis of input conditions are:

Opening of the HV cabin door by the MGB door module requires:

- key transfer system: key is NOT in NBI control room, key is in HV cabin lock in the torus hall,

- confirmation that the high voltage signals are safely offline,

- switch off of the power from the control system for the short-circuit-switches immobilizing them. The switches are automatically closed by gravity,

- confirmation via position monitoring that all short-circuit-switches are safely in the closed position,

- RF generators are in EMERGENCY STOP mode (exclusion of hazards by RF power).

Utilization of HV control signals and the opening of the short-circuit-switches requires:

- authorization for use of high voltage from the W7-X safety controller (cSS),

- HV cabin must be closed and locked determined by the MGB module. The MGB module is a combination of actuator and sensor,

- key transfer system: HV cabin locked in TH and key in NBI control room

\subsection{Danger of radio frequency ( $1 \mathrm{MHz}$ with $150 \mathrm{KW}$ )}

The radio-frequency generators were manufactured by the company Transradio in 2015/16 and comply with the current European standards. The installation location of the $\mathrm{RF}$ generators is in lockable chain link cages in an access limited room. Approximately $100 \mathrm{~m}$ of RF coaxial cable supplies the respective RF driven ion sources located in the HV cabin.
Personnel hazards are only present in the area of the RF sources. These hazards have been excluded by:

- mechanical construction of the HV cabin; RF-tight for the expected frequencies,

- automatic RF transmitter switch-off at high reflected power levels within microseconds,

- accessibility of the HV cabin severely restricted and part of the safety control system (see previous section "Danger of high voltage"),

- safety control: Opening of the HV cabin door is only possible when the RF generators are switched off or in the EMERGENCY STOP position.

\subsection{Danger from gases $\mathrm{H}_{2} / \mathrm{D}_{2}$}

The hazard to personnel and equipment due to the gases $\mathrm{H}_{2} / \mathrm{D}_{2}$ used to generate the beams extracted from the ion sources occurs if they reach the lower explosion limit (LEL) of $4 \%$. Gas monitoring inside the torus hall (TH) is part of the safety control of W7-X; however, the HV cabin of NBI represents a special case. Due to their light-tight construction, the cabin reduces the air circulation with the $\mathrm{TH}$ environment when the door is closed. It was; therefore, a requirement from the $\mathrm{W} 7-\mathrm{X}$ project to monitor the hydrogen levels of this area by the NBI group. Therefore an additional $\mathrm{H}_{2} / \mathrm{D}_{2}$ gas sensor was installed in the $\mathrm{HV}$ cabin. The associated control unit reports three different states to the NBI safety controller via safety relay contacts, which lead to the following reactions of the NBI safety controller:

\section{- Device error}

$\Rightarrow$ NBI-Emergency-Stop (incl. closing of all gas valves)

- Gas alarm $1\left(\mathrm{H}_{2} / \mathrm{D}_{2}>0.8 \%=20 \% \mathrm{LEL}\right)$

$\Rightarrow$ Beam stop and closing of all gas valves

- Gas alarm $2\left(\mathrm{H}_{2} / \mathrm{D}_{2}>1,6 \%=40 \% \mathrm{LEL}\right)$

$\Rightarrow$ NBI-Emergency-Stop (incl. closing of all gas valves)

Closing all gas valves technically ensures that the explosion limit in the HV cabin cannot be reached due to the small amount of gas in the system at any time. For personnel protection, the HV cabin may be entered after a gas alarm only with a handheld gas warning device. This holds until an authorized person has measured the hydrogen level and authorizes free entry.

During commissioning, the insufficient shielding of the sensor cable between the gas sensor and the associated control unit, installed by a manufacturer-certified company, proved to be a problem. With almost every high voltage breakdown in the ion source, the gas monitoring system triggered a NBI-Emergency-Stop. After eliminating the defect and optimizing the shielding, the gas monitoring system functioned error free for the rest of the operation period.

\subsection{Danger of radiation (X-rays, gamma, neutrons)}

$\mathrm{X}$-ray radiation, independent of the operating gas used, can be generated within the RF sources by the impact of accelerated electrons on the source walls or the ion extraction grids. The RF sources are located inside the HV cabin, so that the immediate environment of the X-ray 
source is safely closed during NBI operation (see section "Danger of high voltage").

In addition, the X-ray radiation is shielded by special constructive measures (extra lead shielding) so that personnel safety is guaranteed at all times outside the closed HV cabin.

The limit value of X-rays is $<1 \mathrm{mSv} /$ year and has been proven by extensive measurements that have been approved by state authorities.

Neutron and gamma, plus tritium contamination is only relevant for NBI deuterium $\left(\mathrm{D}_{2}\right)$ beams, which will only be used as an operating gas at a later point in time. Currently, only pure $\mathrm{H}_{2}$ operation is possible, whereby the resulting non X-ray radiation, due to physical reasons, is smaller than the detection limit. Later NBI operation with deuterium will only be permitted with closed torus hall and requires special approval by the state authorities. All safety measures required for deuterium operation, including recording of radiation exposure, will be part of the W7-X safety control system.

\subsection{Danger of failure of cooling}

The NBI cooling water systems operated with pressures up to 16 bar. The system is designed and manufactured in accordance with the Pressure Equipment Directive 97/23/EC (PED) and is operated in accordance with the current German version of Directive 2009/104/EC (BetrSichV). The application of these special standards/directives ensures that personnel hazards are minimized.

The NBI cooling water system is currently only integrated into the NBI safety controller via the NBI-EMERGENCY STOP. In the event of an EMERGENCY STOP, all power supplies of the cooling system are switched off plus the flaps and ball valves of all main lines are closed, minimizing all hazards and reducing possible water leakage. In OP1.2, only the standard control system monitored that the cooling water flow rate was sufficient in all components. Integration into the NBI safety controller is planned for OP2.

\subsection{Danger of $\mathrm{H}_{2} / \mathrm{D}_{2}$-Beam}

$\mathrm{H}_{2} / \mathrm{D}_{2}$ beams can cause high system damage due to their high power densities $\left[\sim 40 \mathrm{MW} / \mathrm{m}^{2}\right]$ either due to insufficient cooling or incorrect operating parameters. Beams cannot spread into air, therefore personnel hazards can only be expected in connection with materials heated by the beam.

In OP1.2 the monitoring of the beam dump in the W7-X vessel with a safety level of SIL2 became necessary due to the possible high repair costs and long downtime of the W7-X if in accident occurs.

The heat shield thermography (HST) is safety diagnostic to prevent damage to the $\mathrm{CuCrZr}$ cooling structure of the NBI beam dump area inside the W7-X vessel. A pyrometer detects if the graphite tile temperature is above a set temperature $\left(\sim 900^{\circ} \mathrm{C}, \mathrm{KiP}\right.$ calculations [10]) and activates the termination of the beam. Each NBI beam is monitored by a separate pyrometer.

The HST safety control is designed as a stand-alone solution and integrated into the NBI safety control via safe output signals and the pyrometer outputs. The HST safety controller, also a Pilz PNOZmulti, controls the HST selftests (pre-shot test) and authorizes the NBI beams only if the self-test was successful.. When the HST triggers, the beam is aborted via two different signal paths. The first path blocks the fast HV signals via the logic gates of the fast interlock system. The second path is via the safety control system: the HV signal path is blocked and the power output of the RF transmitter are switched off by means of safety contacts (see Fig.2).

Although the HST pyrometers and the safety controller meet the SIL3 failure probability requirements, the HST's single-channel architecture usually achieves a safety level of SIL 1 [7]. To comply with SIL2, additional thermocouple-based temperature monitoring of the affected beam dump areas had to be implemented in the W7-X safety controller.

The HST commissioning was perform with time-limited test beams into the empty W7-X torus. These test beams heated the beam dump to the threshold temperature of the pyrometer, which generated a signal used to shut off the beams via the fast interlock and safety control systems. During later NBI operation in OP $1.2 \mathrm{~b}$ the HST was always active. There was never a beam shutdown; in all subsequent plasma experiments the beam dump temperature was always below the trigger threshold.

\section{Summary and Outlook}

The implemented NBI safety controller (including HST) worked reliably and error-free during the entire OP1.2b after commissioning and validation were completed.

The existing safety analysis has proved sufficient for OP1.2b. As far as personnel hazards are concerned, no improvement for the next experiment campaign (OP2) is required.

In terms of system safety, optimization options for potential damage from $\mathrm{H}_{2} / \mathrm{D}_{2}$ beams were identified. Technical solutions and costs are currently being discussed, this concerns:

- Monitoring of the magnet current as a function of the acceleration voltage. For a given acceleration voltage, a defined current must flow through the NBI deflecting magnet so that the non-neutralized particles are deflected in a defined manner onto the water-cooled ion dumps. In the event of an incorrect magnet current, the non-neutralized particles can hit uncooled surfaces inside the injector and lead to high levels of equipment damage. This has occurred after OP1.2b during a test beam.

- Monitoring of the most important cooling water flow values. This, at present, is part of the standard control system. Due to the high power density of the NBI beams, the beams must be switched off immediately in the case of insufficient water flow in the highly loaded components in order to avoid material damage.

\section{Acknowledgments}

This work has been carried out within the framework of the EUROfusion Consortium and has received funding from the Euratom research and training program 20142018 and 2019-2020 under grant agreement number 
633053. The views and opinions expressed herein do not necessarily reflect those of the European Commission.

\section{References}

[1] D. Naujoks, „Sicherheitsanalyse der W7-X Komponenten" internal documentation of W7-X: 1-NBF-V0005.3 [2016]

[2] D. Naujoks, „Bewertung von Ausfallszenarien der Anlage W7-X“ internal documentation of W7-X: 1-NBF-T2007.2 [2014]

[3] D.A. Hartmann, „Sicherheitsanalyse der NBI für OP1.2“; internal documentation of W7-X: 1-CD-T0002 [2018]

[4] Functional safety - Safety instrumented systems for the process industry sector - Part 3: Guidance for the determination of the required safety integrity levels (IEC 61511-3:2003+ Corrigendum 2004); German version EN 61511-3:2004

[5] Safety of machinery - General principles for design - Risk assessment and risk reduction (ISO 12100:2010); German version EN ISO 12100:2010

[6] Machinery Directive, Directive 2006/42/EC of the European Parliament and of the Council of 17 May 2006

[7] S. Degenkolbe „Einfluss der Ausfallwahrscheinlichkeit der HST auf die Gesamtausfallwahrscheinlich für die Abschaltung eines NBI-Beams"; internal documentation of W7-X: 1NBF-T8003.0 [2017] 